Aus der Universitäts-Kinderklinik in Freiburg i. Br. (Direktor: Prof. C. T. Noeggerath.)

\title{
Senföl in Bolus alba statt Senfwickel und Schmierseifeeinreibungen.
}

Von Dr. Albrecht Mertz, Assistent.

$\mathrm{Zu}$ den leider so zahlreichen Mitteln, die wir infolge des Krieges und der Blockade entbehren mußten und noch müssen, gehört auch das Senfmehl. Sein Mangel wird gerade dem Kinderarzte oft genug fühlbar, da seine seit langem bewăhrte vorzügliche Wirkung in Gestalt der Heubnerschen Senfeinwicklung bei der kindlichen Bronchiolitis und auch Pneumonie kaum ersetzlich ist.

Das wirksame Prinzip der Senfpackung sind die durch hydrolytische Spaltung im Wasser freiwerdenden Senföle, die die erwūnschte starke Hyperämie der Haut und damit Ableitung hervorrufen. Das Senföl selbst - das Isosulfozyanallyl - ist aber synthetisch darstellbar und nach wie vor als solches erhältlich.

Um es in einer der Wirkung des Senfwickels gleichsinnigen Weise anwenden zu können, mußte das, unverdünnt schwerste Blasen- und Nekrosenbildung verursachende Senföl in entsprechendem Maße verdünnt und in einem zweckmäßigen Vehikel auf die Haut aufgetragen werden.

Auf Veranlassung von Herrn Prof. Noeggerath unternahm ich es, eine Verdünnung des Senföles mit Bolus alba herzustellen und als Senfmehlersatz auszuprobieren. Die Verdünnung, an der eigenen Haut austitriert, hat sehr weitgehend zu sein. Als der natürlichen Senfmehlwirkung etwa gleichkommend erwies sich eine Konzentration von 3-4 Tropfen reinen Senföles in $100 \mathrm{~g}$ Bolus alba.

Die praktische Anwendung gestaltet sich folgendermaßen: Wir mischen die nach der Größe.des Kindes einzurichtende Bolusmenge, die für Säuglinge etwa $200-300 \mathrm{~g}$, für größere Kinder $400-800 \mathrm{~g}$ beträgt, auf je $100 \mathrm{~g}$ mit 3-5 Tropfen Senföl durch sehr sorgfältiges Umrühren, geben etwa vier Fünftel des Bolusgewichtes an heißem Wasser hinzu und streichen den gleichmäßig verrührten Brei rasch auf den der Kindergröße angepaßten Wickel aus, in dem das Kind, ganz entsprechend dem Vorgehen bei der natürlichen Senfmehleinwicklung, eingeschlagen wird. Ein zweites, am besten wollenes Tuch schließt dicht $a b$, besonders ist auch hier auf die Abdichtung am Halse durch ein feuchtes. Taschen- 
tuch zu achten, um Reizung von Augen und Atmungswegen zu vermeiden. Nach 10-15 Minuten kommt das Kind in das bereitgehaltene Reinigungsbad, in dem sich der Bolusbrei ohne Schwierigkeit rasch abwaschen läßt. Das Kind ist bei gelungener Prozedur krebsrot und bekommt nun, auch ganz gemä $B$ der alten $H e$ u bnerschen Vorschrift, noch ein heißes Bad und anschließende Schwitzpackung, deren Dauer nach dem Zustande des Herzens bemessen wird und $1 / 2-1-2$ Stunden beträgt. Der Senfölbolusbrei muß kurz vor dem Gebrauch hergestellt werden; er hält sich nicht, weil das Senföl sich alsbald verflüchtigt.

Die Indikationen und Erfolge unseres Verfahrens sind ganz die der natürlichen Senfmehleinwicklung. Es besitzt darüber hinaus und im Gegensatz zu dieser noch den großen Vorzug der Dosierbarkeit. Für die Săuglinge wählen wir die geringere Konzentration von 3 Tropfen auf $100 \mathrm{~g}$ Bolus, für die älteren Kinder nehmen wir 4 Tropfen und eventuell auch $41 /-5$, wenn uns an einer besonders raschen und energischen Wirkung liegt. Für exsudative Säuglinge mit sehr reizbarer Haut gehen wir sogar auf 2 Tropfen herunter und lassen dafür den Wickel etwas länger liegen.

Eine übermäßig lang anhaltende oder schädliche Reizung der Haut trat nie in Erscheinung. Nach zwei Stunden ist die Rötung gewöhnlich wieder verschwunden, höchstens finden sich am folgenden Tage noch Spuren. Im Selbstversuche verspürt man bei der Konzentration von 4 Tropfen auf $100 \mathrm{~g}$ anfangs ein leichtes Brennen der Haut, das nach einigen Minuten auftritt und dann sehr rasch von einem nicht unangenehmen, lediglich bestehenbleibenden Wärmegefühl abgelöst wird. Auch werden selbst solche Kinder, die anfangs unruhig sind oder gar etwas schreien, dementsprechend in der Packung sehr bald wieder ruhig und nehmen ihre Flasche.

Anhangsweise darf ich noch berichten, daß wir unsere SenfölBolusmischung auch als Ersatz von Einreibungen mit der gleichfalls nicht mehr erhältlichen Schmierseife bei der Behandlung der Drüsentuberkulose versucht haben. Wegen der hierbei beabsichtigten milderen Wirkung wählten wir eine Konzentration von 1 Tropfen auf $100 \mathrm{~g}$ und bedeckten in der Art einer Schmierkur abwechselnd obere und untere Extremitäten. Brust und Rücken mit dem Bolusbrei, der dann etwa zwei Stunden liegen blieb. Auch zu Einreibungen des Bauches bei Tuberkulose der Bauchorgane kann er verwendet werden; irgendeine unangenehme Hautreizwirkung tritt auch dabei nicht, ein. Ueber den Erfolg des ietzteren Verfahrens können wir wegen der zu geringen Anzahl der bisher behandelten Fälle und der Kürze unserer Versuchsdauer noch nicht abschìießend urteilen.

Wir glauber, dạ $B$ der Senfbolusbréi seiner bequemen Herstellung und exakten Dosierbarkeit wegen auch dann noch für die Behandlung akuter Lungenerkrankungen als Senfbreiersatz und in der Tuberkulosetherapie an Stelle der Schmierseife Geltung behalten wird, wenn diese Mittel wieder erhältlich sind. 